Performance of the ATLAS detector and its electronics under first beam conditions

This article has been downloaded from IOPscience. Please scroll down to see the full text article.

2010 JINST 5 C12011

(http://iopscience.iop.org/1748-0221/5/12/C12011)

View the table of contents for this issue, or go to the journal homepage for more

Download details:

IP Address: 137.138.125.164

The article was downloaded on 10/01/2011 at 10:27

Please note that terms and conditions apply. 
Topical Workshop on Electronics for Particle Physics 2010, 20-24 SEPTEMBER 2010, AACHEN, GERMANY

\section{Performance of the ATLAS detector and its electronics under first beam conditions}

ATLAS collaboration

\section{T. Pauly}

CERN,

Geneva, Switzerland

E-mail: Thilo.Pauly@cern.ch

ABSTRACT: Since spring 2010 the LHC delivers proton-proton collisions at a centre-of-mass energy of $7 \mathrm{TeV}$, marking the start of its high-energy physics program. In this paper, we give an overview of the ATLAS detector during this period, with emphasis on the performance of the subdetectors and their electronics. We cover operational aspects necessary for smooth, safe, and stable data taking, as well as discuss resolved and pending electronics issues.

KEYWORDS: Trigger concepts and systems (hardware and software); Detector control systems (detector and experiment monitoring and slow-control systems, architecture, hardware, algorithms, databases); Data acquisition concepts 


\section{Contents}

1 Introduction 1

2 Detector status and data taking 1

3 Timing issues 3

4 Detector performance $\quad 4$

5 Summary 5

\section{Introduction}

In this paper, we give an overview on the performance of the ATLAS detector [1] with first LHC beams until September 2010. The first part describes the status of the detector and operational aspects of data taking. The second part is concentrating on timing issues in connection with detector operation. In the last part, we show a few examples of recent studies that document the performance of the detector.

\section{Detector status and data taking}

The status of the ATLAS detector in 2010 (until June) is summarised in table 1, where one can see, for each sub-detector, the number of channels, followed by the approximate fraction of channels operational, which is larger than $97 \%$ for each sub-system. While the overall detector is performing well, there are a few issues with component failures, that are currently under investigation [2].

Figure 1 (left) shows the ATLAS online luminosity versus time for a typical fill, starting with a luminosity of $2.5 \times 10^{30} \mathrm{~cm}^{-2} \mathrm{~s}^{-1}$. The luminosity is shown when the LHC operators declare the beams stable, at which time the high-voltage of the silicon tracking detectors and the muon systems are ramped up. This process takes a few minutes and the recorded luminosity after this time is shown overlaid. The overall efficiency is very close to $100 \%$, although a few short dips can be seen in the recorded luminosity, where deadtime was applied, typically due to operational sub-detector failures, which are recovered within seconds or minutes. The last column of table 1 lists the luminosity-weighted relative fraction of detector uptime and good quality data delivered during LHC fills with stable beams at a centre-of-mass energy of $7 \mathrm{TeV}$, between March 30th and August 30th, 2010. This fraction stays above $93 \%$ for each sub-detector. 
Table 1. Overview of the status of the ATLAS sub-detectors. The 'good for physics' fraction in the last column is defined as the luminosity-weighted relative fraction of detector uptime and good quality data delivered during LHC fills with stable beams at a centre-of-mass energy of $7 \mathrm{TeV}$, between March 30th and August 30th, 2010.

\begin{tabular}{|lccc|}
\hline Sub-detector & $\begin{array}{c}\text { Number of } \\
\text { channels }\end{array}$ & $\begin{array}{c}\text { Operational } \\
\text { channels in \% }\end{array}$ & $\begin{array}{c}\text { 'Good for physics' } \\
\text { fraction in \% }\end{array}$ \\
\hline Pixels & $80 \mathrm{M}$ & 97.3 & 96.7 \\
SCT Silicon Strips & $6.4 \mathrm{M}$ & 99.2 & 97.5 \\
TRT Transition Rad. Tracker & $350 \mathrm{k}$ & 97.1 & 100.0 \\
\hline LAr EM Calorimeter & $170 \mathrm{k}$ & 98.1 & 93.8 \\
Tile Calorimeter & 9800 & 96.9 & 99.7 \\
Hadronic Endcap LAr Calorimeter & 5600 & 99.9 & 98.8 \\
Forward LAr Calorimeter & 3500 & 100.0 & 99.0 \\
Level-1 Calo Trigger & 7160 & 99.9 & - \\
\hline Level-1 Muon RPC Trigger & $370 \mathrm{k}$ & 99.5 & - \\
Level-1 Muon TGC Trigger & $320 \mathrm{k}$ & 100.0 & - \\
MDT Monitored Drift Tubes & $350 \mathrm{k}$ & 99.7 & 98.6 \\
CSC Cathode Strip Chambers & $31 \mathrm{k}$ & 98.5 & 98.5 \\
RPC Barrel Muon Chambers & $370 \mathrm{k}$ & 97.0 & 98.6 \\
TGC Endcap Muon Chambers & $320 \mathrm{k}$ & 98.6 & 98.5 \\
\hline
\end{tabular}
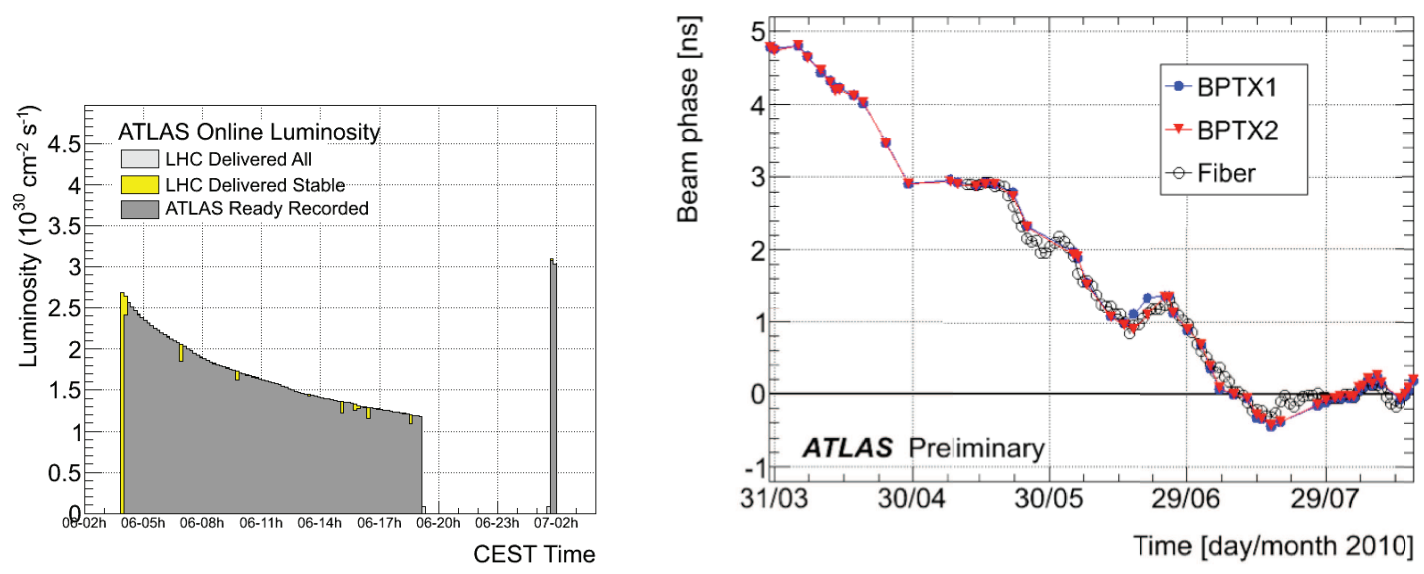

Figure 1. Left: example of a typical LHC fill, showing the instantaneous luminosity as a function of time. Right: beam timing with respect to the LHC $40 \mathrm{MHz}$ clock measured by the ATLAS beam timing pick-ups (BPTX) for beam 1 and beam 2 in spring and summer 2010. A clock fiber propagation delay measurement is overlaid. 


\section{Timing issues}

In this section, we highlight two electronics issues related to the stability of the $40 \mathrm{MHz}$ bunch clock received by the LHC. This clock is distributed to all sub-detectors and typically used for digitisation of the analog signals and for serial data transmission off the detector, both of which require good phase stability. This is accomplished by series of phase-locked loops, in which the QPLL chip [3] typically plays a critical role. In ATLAS, there is a hierarchy of QPLLs, with a top-level QPLL in the LHC timing receiver board (RF2TTC [4]) followed by trees of QPLLs per sub-detector with depths ranging from 0 (no QPLL used) up to 3. It is important that all QPLLs stay locked during data-taking, otherwise data corruption, readout inefficiencies or deadtime can occur. This would be assured by a stable LHC $40 \mathrm{MHz}$ bunch clock. But the LHC clock is not entirely stable: the acceleration ramp causes smooth and slow frequency changes of tens to hundreds of $\mathrm{Hz}$, sudden jumps in frequencies can also arise, for instance during special LHC measurements (chromaticity, loss maps), during the LHC setup procedure, and during the ATLAS setup procedure. ATLAS data-taking is potentially affected by these clock instabilities, as data taking typically starts well in advance of LHC fills.

Several improvements have been undertaken in the operational procedure to cope with these instabilities, in the configuration process of the QPLLs, as well as in recovery procedures. For a QPLL to operate stably without unlocking at a suitable working point, it has to be reset while receiving a clock with a well-defined frequency value. This clock is provided by an internally generated clock, currently set to the frequency corresponding to protons at injection. Using this clock, all QPLLs are being reset in the correct order, from top to bottom. Where possible, the reset is performed directly via the reset pin, though in some cases the electronics does not allow for this and an indirect method has to be applied: when a QPLL is operating in automatic mode, a forced unlock will initiate a calibration procedure of the chip, which is equivalent to a reset. Two options have been explored to forcefully unlock the QPLL: a large enough phase jump induced by the on-detector timing receiver chips, and a frequency jump by $1 \mathrm{MHz}$. While the former seemed to work well, the latter does not give satisfactory results and is being replaced by the phase jump method. In order to cope with unlocking QPLLs during data-taking, some sub-detectors have implemented recovery procedures that allow a re-synchronisation of the failing detector part within a few seconds $[5,6]$.

A second issue being addressed is an observed drift of the measured phase between the bunch arrival times and the LHC clock. Figure 1 (right) shows the phase measurements over time for each of the two beams, using a monitoring system based on beam timing pick-ups (BPTX) [7]. A drift of more than $5 \mathrm{~ns}$ is observed between April and July 2010. This is consistent with an increase of the propagation delay in the $13.3 \mathrm{~km}$ long optical fiber that transports the timing signals from the RF system at LHC Point-4 to ATLAS. In order to test this hypothesis, a measurement of the propagation delay of spare fibers that share part of the propagation path is overlaid, after being scaled appropriately. The propagation delay changes nicely follow the beam timing measurements, proving therefore that the drift stems from the clock propagation rather than from a drift of the beams. In order to provide stable beam timing within $\pm 500 \mathrm{ps}$, corrective measures are being performed regularly using the fine-delay in the RF2TTC module with a minimum step size of 500 ps. 

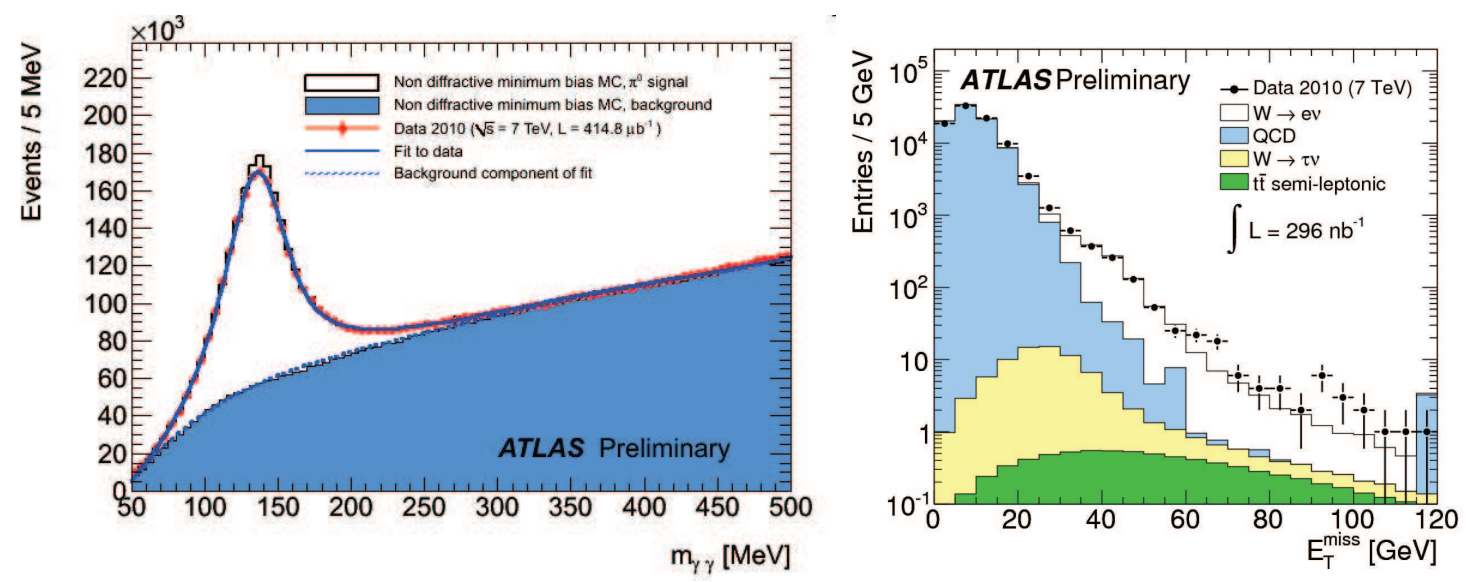

Figure 2. Left: di-photon invariant mass for data and Monte Carlo simulation. The simulation is normalized to the same number of entries as the data. Right: distribution of the missing transverse energy of $\mathrm{W} \rightarrow$ e $v$ candidates for data and Monte Carlo simulation, broken down into the signal and various background components.

\section{Detector performance}

Lots of progress has been made in understanding the detector performance, employing more and more data-driven methods in addition to comparisons between data and simulation. In this section, we give a few examples of such recent studies.

Good progress has been made in understanding the performance of the tracking. The alignment of the Inner Detector is already close to ideal. The track momentum scale has been studied, using decays of a range of well-established resonances, from the relatively simple $\mathrm{K}_{\mathrm{S}}^{0} \rightarrow \pi^{+} \pi^{-}$ decays, to more complicated cascade decays, such as $\Omega(1670) \rightarrow \Lambda K$. From these studies, the momentum scale at low transverse momentum is known to better than a percent. The material distribution is mapped using $\gamma$ conversions and secondary hadronic interactions and agrees with the Monte Carlo simulation at the $10 \%$-level. Further studies aim at achieving a material description at the percent level.

Using a sample of $\pi^{0} \rightarrow \gamma \gamma$ decays (see figure 2 left) allowed to study the response uniformity of the electromagnetic calorimeter. From the reconstructed mass ratio of data to Monte Carlo, the response uniformity was found to be better than $\pm 2 \%$ along the full pseudo-rapidity range and in azimuth.

Good understanding of missing energy will be crucial for new physics discoveries. Using minimum bias events, the missing transverse energy spectrum was verified to agree with expectations over 6 orders of magnitude ranging to missing transverse energies of up to $60 \mathrm{GeV}$. The missing energy resolution in $x$ and $y$ as a function of the transverse energy sum $E_{T}^{\text {sum }}$ was determined as $0.49 \cdot \sqrt{E_{T}^{\text {sum }}}$, in agreement with the Monte Carlo expectation. The right plot of figure 2 shows the transverse missing energy distribution of the $\mathrm{W} \rightarrow \mathrm{e} v$ candidates from a sample of $296 \mathrm{nb}^{-1}$, agreeing very well with the Monte Carlo expectation.

The energy scale of jets measured in the calorimeters is calibrated on average to the hadronic 

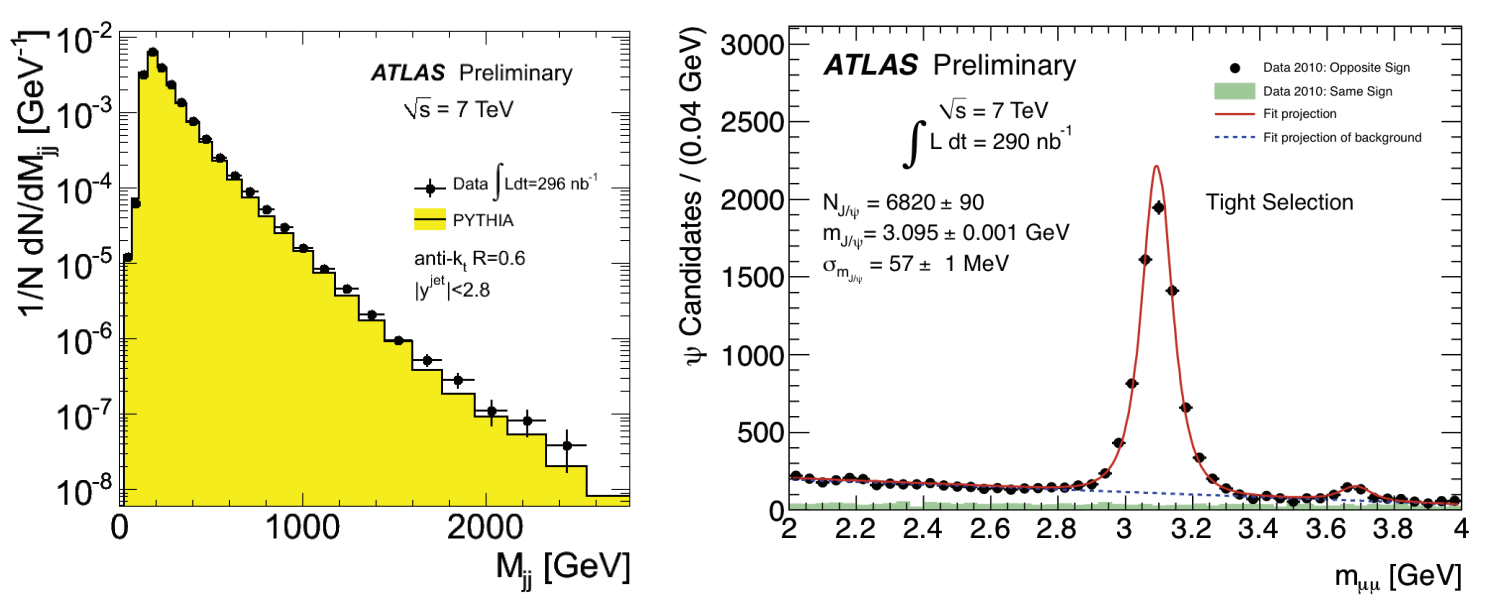

Figure 3. Left: reconstructed mass spectrum for high-energy di-jets, from data and a Monte Carlo Simulation. Right: di-muon spectrum of $J / \psi$ and $\psi(2 S)$ candidates passing vertexing with invariant masses between 2 and $4 \mathrm{GeV}$. The signal lineshape fits are both Gaussian with a second-order polynomial to model the background.

scale using a method based on a simulated Monte Carlo QCD jet sample, which has been validated with data collected at $\sqrt{s}=900 \mathrm{GeV}$ and $7 \mathrm{TeV}$. The systematic uncertainties on the jet energy scale is evaluated by comparing the nominal results to Monte Carlo simulations using alternative detector configurations, hadronic shower and physics models, and by comparing the relative response of jets across pseudo-rapidity between data and simulation. For inclusive jets with transverse momentum greater than $20 \mathrm{GeV}$ and pseudo-rapidity smaller than 2.8 , the jet energy scale is determined with an uncertainty of smaller than $10 \%$. Figure 3 (left) shows a di-jet reconstructed mass spectrum for high-energy jets, which agrees with the simulation over 6 orders of magnitude. The spectrum reaches beyond $1.96 \mathrm{TeV}$, therefore probing a new region of phase space not accessible to the Tevatron.

Reconstructing the decay of known resonances such as $J / \psi \rightarrow \mu \mu$ is an important benchmark for the performance of the muon system. Figure 3 (right) shows the invariant mass distribution of reconstructed $J / \psi \rightarrow \mu^{+} \mu^{-}$candidates using both the muon system and the Inner Detector. Using this sample, the momentum resolution of low-momentum muons is estimated to be around $2 \%$ and the absolute momentum scale to better than $0.2 \%$.

\section{Summary}

The first few months of data taking with the ATLAS detector with LHC collisions at $7 \mathrm{TeV}$ have yielded a wealth of data of good quality. All sub-detectors are working very well, with good data quality and a data taking efficiency of better than $94 \%$. There is good and rapid progress in the understanding of the detector performance, employing more and more data-driven methods in addition to the comparisons between data and simulation. 


\section{References}

[1] ATLAS collaboration, G. Aad et al., The ATLAS Experiment at the CERN Large Hadron Collider, 2008 JINST 3 S08003 [SPIRES].

[2] T. Flick, VCSEL failures in ATLAS, in Topical Workshop on Electronics for Particle Physics 2010, Aachen, Germany, 20-24 September 2010.

[3] P. Moreira, QPLL Manual, http://proj-qpll.web.cern.ch/proj-qpll/.

[4] S. Baron, TTC challenges and upgrade for the LHC, Proceedings of 11th Workshop on Electronics for LHC and Future Experiments, Heidelberg Germany (2005), pp 125-129.

[5] D. Olivito, Performance of the ATLAS Transition Radiation Tracker read-out with cosmic rays and first high energy collisions at the LHC, in Topical Workshop on Electronics for Particle Physics 2010, Aachen, Germany, 20-24 September 2010.

[6] T. Hayakawa, Detailed Performance Study of the ATLAS Endcap Muon Trigger with Beam Collision Data, in Topical Workshop on Electronics for Particle Physics 2010, Aachen, Germany, 20-24 September 2010.

[7] C. Ohm and T. Pauly, The ATLAS beam pick-up based timing system, Nucl. Instr. and Meth. A 623 (2010) 558 [arXiv: 0905.3648v1]. 